\title{
Nonequilibrium spin-dependent phenomena in mesoscopic superconductor-normal metal tunnel structures
}

\author{
Francesco Giazotto, ${ }^{1, *}$ Fabio Taddei, ${ }^{1}$ Pino D'Amico, ${ }^{1,2}$ Rosario Fazio, ${ }^{1,3}$ and Fabio Beltram ${ }^{1}$ \\ ${ }^{1}$ NEST CNR-INFM and Scuola Normale Superiore, I-56126 Pisa, Italy \\ ${ }^{2}$ Institut für Theoretische Physik, Universität Regensburg, D-93040 Regensburg, Germany \\ ${ }^{3}$ International School for Advanced Studies (SISSA), I-34014 Trieste, Italy
}

(Received 1 June 2007; revised manuscript received 27 August 2007; published 20 November 2007)

\begin{abstract}
We analyze the broad range of spin-dependent nonequilibrium transport properties of hybrid systems composed of a normal region tunnel coupled to two superconductors with exchange fields induced by the proximity to thin ferromagnetic layers and highlight its functionalities. By calculating the quasiparticle distribution functions in the normal region, we find that they are spin dependent and strongly sensitive to the relative angle between exchange fields in the two superconductors. The impact of inelastic collisions on their properties is addressed. As a result, the electric current flowing through the system is found to be strongly dependent on the relative angle between exchange fields, giving rise to a huge value of magnetoresistance. Moreover, the current presents a complete spin polarization in a wide range of bias voltages, even in the quasiequilibrium case. In the nonequilibrium limit, we parametrize the distributions with an "effective" temperature, which turns out to be strongly spin dependent, though quite sensitive to inelastic collisions. By tunnel coupling the normal region to an additional superconducting electrode, we show that it is possible to implement a spin-polarized current source of both spin species, depending on the bias voltages applied.
\end{abstract}

DOI: $10.1103 /$ PhysRevB.76.184518

PACS number(s): 72.25.-b, 85.75.- d, 74.50.+r, 05.70.Ln

\section{INTRODUCTION}

Although the interest in nonequilibrium superconductivity dates back to the $1970 \mathrm{~s},{ }^{1}$ nonequilibrium transport phenomena in hybrid superconducting structures are currently under the spotlight. One of the key experiments that renewed this interest was probably the control of the supercurrent flowing through a Josephson junction, and even the reversal of its sign, accessible by altering the quasiparticle population in the weak link (see Ref. 2 and references therein). Out-ofequilibrium electron population can be realized in mesoscopic conductors subject to a bias voltage in which electrons cannot exchange energy either with one another or with lattice phonons, so that their energy distribution is not Fermi-like. ${ }^{3}$ Quasiequilibrium is reached if electrons can thermalize, while still decoupled from the phonons, so that they can reach a temperature which is different from the one relative to the phonon bath. In ballistic Josephson junctions, supercurrent control occurs by inducing a nonequilibrium population of Andreev levels either by injecting a current through an additional normal terminal connected to the weak link $^{4,5}$ or by applying an electromagnetic radiation on the weak link. 6,7 The diffusive long-junction limit was considered $^{8-11}$ and experimentally realized too. ${ }^{12,13}$ The control of supercurrent by cooling electrons in the weak link was proposed in Refs. $14-17$ and experimentally realized. ${ }^{18}$ It is worthwhile stressing that electron temperature can be lowered below the phonon temperature, thus realizing electron microrefrigeration, ${ }^{19,20}$ by exploiting the superconducting energy gap (see Refs. 21 and 22 and references therein).

Spin-dependent properties in out-of-equilibrium hybrid systems were investigated in a limited number of articles. In Refs. 23-26, ferromagnet-superconductor-ferromagnet double tunnel junctions were considered in order to study the spin imbalance induced in $\mathrm{S}$ by nonequilibrium. In the anti- ferromagnetic alignment of the magnetizations of the F layers, a strong suppression of superconductivity was found, leading to a large magnetoresistive effect. In Josephson junctions, the effect of spin injection ${ }^{27}$ and the presence of weak ferromagnets $^{28}$ were considered, while the effect of the Andreev reflection on spin accumulation in a ferromagnetic wire was reported in Ref. 29. In Ref. 30, the possibility of manipulating magnetism through the interplay of superconductivity and nonequilibrium transport was investigated. Recently, we have proposed ${ }^{31}$ a hybrid ferromagnetsuperconductor (FS) spin valve whose operation is based on the interplay between out-of-equilibrium quasiparticle dynamics and proximity-induced exchange coupling in superconductors. Huge tunnel magnetoresistance values as high as several $10^{6} \%$ has been predicted, leading to a fully tunable structure which shows high potential for application in spintronics. In this paper, we comprehensively investigate the physics and functionality of the setup analyzed in Ref. 31, extending our study to the presence of finite electronelectron interaction and to the quasiequilibrium limit, as well as to the presence of nonidealities in the superconductors. In this setup, a spin-dependent "effective" temperature for the electrons in the $\mathrm{N}$ region emerges, thus leading to possible spin-dependent thermoelectric effects.

The paper is organized as follows. In Sec. II, we describe the system under investigation, and in Sec. III, we derive the quasiparticle distribution functions in different regimes. In particular, we consider the nonequilibrium limit in Sec. III A, we include the effect of inelastic collisions in Sec. III B, and we describe the quasiequilibrium regime in Sec. III C. In Sec. IV, we discuss the behavior of the electric current, focusing on the magnetoresistive effects and on the spinfiltering properties of the system in Sec. V. Section VI is devoted to the characterization of the nonequilibrium distribution through an "effective" temperature and to the exploi- 

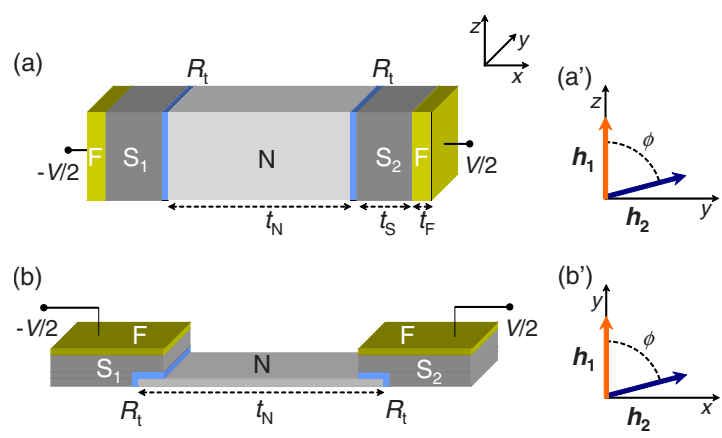

FIG. 1. (Color online) Sketch of two possible implementations of the FS-I-N-I-SF structure analyzed in this work. (a) Spin-valve setup consisting of a sequence of stacked metallic layers. (b) A planar structure. Ferromagnetic layers $(F)$ induce in each superconductor, through the proximity effect, an exchange field $\left(\boldsymbol{h}_{1,2}\right)$ whose relative orientation can be controlled by an externally applied magnetic field. The $\mathrm{F}$ exchange fields are confined $\left(\mathrm{a}^{\prime}\right)$ to the $y$ - $z$ plane for the setup shown in (a) and $\left(b^{\prime}\right)$ to the $x-y$ plane for the setup shown in (b) and are misaligned by an angle $\phi$. A voltage bias $V$, applied across the structure, allows to control the energy distributions in the $\mathrm{N}$ region. The structure is assumed quasi-one-dimensional.

tation of the system as a source of spin-polarized current through the introduction of an additional superconducting electrode. Finally, we draw our conclusions in Sec. VII.

\section{SETUP}

We consider a device consisting of two identical FS bilayers $\left(\mathrm{FS}_{1,2}\right)$ symmetrically connected to a mesoscopic normal metal region $(\mathrm{N})$ of length $t_{\mathrm{N}}$ through tunnel contacts (I) of resistance $R_{t}$. The concentration of impurities is such that quasiparticle transport is diffusive. The resulting system, a FS-I-N-I-SF heterostructure, is shown in Fig. 1 in two distinct experimental implementations. Figure 1(a) shows a spin-valve-like structure, which consists of a sequence of stacked metallic layers, while Fig. 1(b) displays a planar system. Although the two implementations are equivalent on theoretical footing, the planar configuration allows the measurement of local properties (e.g., the quasiparticle distribution functions as well as the local temperature) by connecting the $\mathrm{N}$ region to additional metallic probes. This will be addressed in Sec. VI. For the sake of simplicity, we assume a symmetric system (a resistance asymmetry would not change the overall physical picture), $t_{\mathrm{F}}\left(t_{\mathrm{S}}\right)$ labels the $\mathrm{F}(\mathrm{S})$ layer thickness and a bias voltage $V$ is applied across the structure. The exchange field in the left ferromagnet $\left(\boldsymbol{h}_{1}\right)$ is aligned along the $z$ axis for the setup in Fig. 1(a) or along the $y$ axis for the setup in Fig. 1(b), while that in the right F layer $\left(\boldsymbol{h}_{2}\right)$ is misaligned by an angle $\phi$ [see Figs. $1\left(\mathrm{a}^{\prime}\right)$ and $\left.1\left(\mathrm{~b}^{\prime}\right)\right]$. For simplicity, we set $\left|\boldsymbol{h}_{1}\right|=\left|\boldsymbol{h}_{2}\right|=h$. In real structures, $\boldsymbol{h}_{2}$ can be rotated by applying an in-plane magnetic field as low as some millitesla. Moreover, we assume that (i) the FS interface is transparent and (ii) $R_{\mathrm{t}}$ is much larger than both the resistance of the $\mathrm{N}$ layer $\left(R_{\mathrm{N}}\right)$ and the FS contact resistance. The first condition ensures that the superconductor is strongly affected by the proximity of the F layer, ${ }^{32}$ while the second ensures that all the voltage drop occurs at the tunnel barriers (so that any spatial variation of the chemical potential within the $\mathrm{N}$ region can be neglected) and that each $\mathrm{FS}$ bilayer is in local equilibrium.

The electronic properties of a FS bilayer can be analyzed within the quasiclassical Green's function formalism. ${ }^{32} \mathrm{We}$ are interested in the situation in which the influence of the F layer on the superconductor becomes nonlocal. This occurs in the limit $t_{\mathrm{S}}<\xi_{\mathrm{S}}=\sqrt{\hbar D / 2 \pi k_{B} T_{c}}$ and $t_{\mathrm{F}}<\xi_{\mathrm{F}}=\sqrt{\hbar D / h}$, where $\xi_{\mathrm{S}}$ and $\xi_{\mathrm{F}}$ are the superconducting coherence length and the length of condensate penetration into the ferromagnet, respectively. $D$ denotes the diffusion coefficient, $T_{c}$ is the superconducting critical temperature, and $k_{B}$ is the Boltzmann constant. In this situation, the ferromagnet induces in $\mathrm{S}$ a homogeneous effective exchange field (analogous to the one present in magnetic superconductors ${ }^{32}$ ) through proximity effect and modifies the superconducting gap $(\Delta)$. The effective values of the exchange field $\left(h^{*}\right)$ and gap $\left(\Delta^{*}\right)$ are given by ${ }^{33}$

$$
\begin{aligned}
& \Delta^{*} / \Delta=\nu_{\mathrm{S}} t_{\mathrm{S}}\left(\nu_{\mathrm{S}} t_{\mathrm{S}}+\nu_{\mathrm{F}} t_{\mathrm{F}}\right)^{-1}, \\
& h^{*} / h=\nu_{\mathrm{F}} t_{\mathrm{F}}\left(\nu_{\mathrm{S}} t_{\mathrm{S}}+\nu_{\mathrm{F}} t_{\mathrm{F}}\right)^{-1},
\end{aligned}
$$

where $\nu_{\mathrm{S}}\left(\nu_{\mathrm{F}}\right)$ is the normal-state density of states (DOS) in $\mathrm{S}$ (F). In particular, if $\nu_{\mathrm{F}}=\nu_{\mathrm{S}}$ and for $t_{\mathrm{F}} \ll t_{\mathrm{S}}$, it follows that

$$
\begin{gathered}
\Delta^{*} / \Delta \simeq 1, \\
h^{*} / h \simeq t_{\mathrm{F}} / t_{\mathrm{S}} \ll 1,
\end{gathered}
$$

i.e., $h^{*}$ turns out to be much smaller than in an isolated $\mathrm{F}$ layer. As a matter of fact, $h^{*}$ can take values of the order of magnitude of $\Delta^{*}$. These conditions can be achieved quite easily in a realistic structure. We assume that the only effect of $h^{*}$ on the quasiparticles is to lead to a spin-dependent superconducting DOS, i.e., we neglect any influence of the induced magnetic moment on the orbital motion of electrons. Furthermore, we assume a negligible spin-orbit interaction. ${ }^{34}$ The superconductor DOS $\left(\mathcal{N}_{\sigma}^{S}\right)$ thus will be BCS-like but shifted by the effective exchange energy (equivalent to that of a Zeeman-split superconductor in a magnetic field ${ }^{35}$ ). By choosing the spin quantization axis along the direction of the exchange field, we have

$$
\mathcal{N}_{\sigma}^{S}\left(\varepsilon, h^{*}\right)=\frac{1}{2}\left|\operatorname{Re}\left[\frac{\varepsilon+\sigma h^{*}+i \Gamma}{\sqrt{\left(\varepsilon+\sigma h^{*}+i \Gamma\right)^{2}-\Delta^{* 2}}}\right]\right|,
$$

where $\varepsilon$ is the energy measured from the condensate chemical potential, $\sigma= \pm 1$ refers to the spin parallel (antiparallel) to the direction of $\boldsymbol{h}_{1}$, and $\Gamma$ is a smearing parameter. ${ }^{36}$ The latter allows quasiparticle states within the gap due to inelastic scattering in the superconductor ${ }^{37}$ or inverse proximity effect from the nearby metallic layers. Typical values for $\Gamma$ lie in the range $\Gamma \sim 1 \times 10^{-5} \Delta, \ldots, 1 \times 10^{-3} \Delta$ for $\mathrm{Al}$ as a thinfilm superconducting electrode. ${ }^{36}$ In the following calculations, we set $\Gamma=10^{-4} \Delta^{*}$, unless differently stated.

In order to describe our system, we make use of the tunneling Hamiltonian approach and neglect proximity effects at NIS interfaces. 


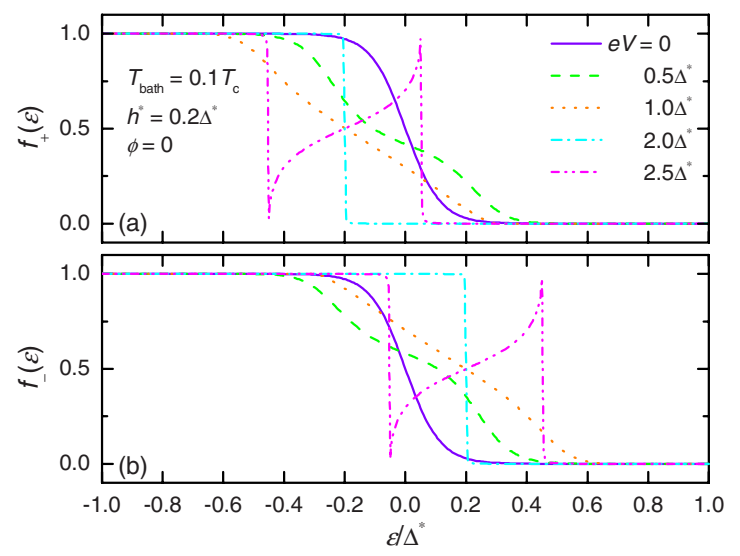

FIG. 2. (Color online) Spin-dependent quasiparticle distribution functions $f_{\sigma}(\varepsilon)$ in the full nonequilibrium limit vs energy $\varepsilon$ for several bias voltages at $\phi=0, T_{\text {bath }}=0.1 T_{c}$, and $h^{*}=0.2 \Delta^{*}$. (a) $f_{+}(\varepsilon)$ and (b) $f_{-}(\varepsilon)$.

\section{QUASIPARTICLE DISTRIBUTIONS}

\section{A. Negligible inelastic scattering: Full nonequilibrium limit}

At finite bias $V$ and in the limit of negligible inelastic scattering, quasiparticles in the $\mathrm{N}$ layer will be out of equilibrium and thus, in general, not distributed according to the Fermi function. The steady-state nonequilibrium distribution functions can be calculated by equating, at each energy value, the tunneling rate of quasiparticle entering the $\mathrm{N}$ region from the insulating layer on the left-hand side to the tunneling rate of those exiting through the right-hand-side barrier. ${ }^{38}$ In the general case of noncollinear exchange fields, the spin eigenstates relative to $S_{2}(|\uparrow\rangle$ and $|\downarrow\rangle)$ can be obtained by rotating the spin eigenstates relative to $S_{1}(|+\rangle$ and $|-\rangle$ ) by the angle $\phi$ (representing the misalignment between $\boldsymbol{h}_{1}$ and $\left.\boldsymbol{h}_{2}\right)$. As a consequence, spin up $(\sigma=+1$ with eigenstate $|+\rangle$ ) quasiparticles exiting the $\mathrm{N}$ layer through the righthand-side barrier will now consist of two contributions. One describes tunneling into spin up (with eigenstate $|\uparrow\rangle$ ) quasiparticles, proportional to $\cos ^{2}[\phi / 2]$, and the other describing tunneling into spin down (with eigenstate $|\downarrow\rangle)$ quasiparticles, proportional to $\sin ^{2}[\phi / 2]$. As a result, the nonequilibrium distribution function in the $\mathrm{N}$ layer is spin dependent and can be written as

$$
f_{\sigma}\left(\varepsilon, V, h^{*}, \phi\right)=\frac{\mathcal{N}_{\sigma}^{S_{1}} \mathcal{F}^{\mathrm{S}_{1}}+\left[a(\phi) \mathcal{N}_{\sigma}^{\mathrm{S}_{2}}+b(\phi) \mathcal{N}_{-\sigma}^{S_{2}}\right] \mathcal{F}^{\mathrm{S}_{2}}}{\mathcal{N}_{\sigma}^{\mathrm{S}_{1}}+a(\phi) \mathcal{N}_{\sigma}^{t \mathrm{~S}_{2}}+b(\phi) \mathcal{N}_{-\sigma}^{\mathrm{S}_{2}}},
$$

where $\quad a(\phi)=\cos ^{2}[\phi / 2], \quad b(\phi)=\sin ^{2}[\phi / 2], \quad \mathcal{F}^{\mathrm{S}_{1}\left(\mathrm{~S}_{2}\right)}$ $=f_{0}(\varepsilon \pm e V / 2), \mathcal{N}_{\sigma}^{\mathrm{S}_{1}}=\mathcal{N}_{\sigma}^{\mathrm{S}}(\varepsilon+e V / 2), \mathcal{N}_{\sigma}^{\mathrm{S}_{2}}=\mathcal{N}_{\sigma}^{\mathrm{S}}(\varepsilon-e V / 2), f_{0}(\varepsilon)$ is the Fermi function at bath temperature $T_{\text {bath }}$, and $e$ is the electron charge.

Figures 2(a) and 2(b) show the nonequilibrium distribution functions [calculated from Eq. (4)] for spin up and spin down quasiparticles, respectively, vs energy $\varepsilon$ for the parallel configuration (i.e., $\phi=0$ ) at $T_{\text {bath }}=0.1 T_{c}, h^{*}=0.2 \Delta^{*}$, and different values of $V$ (we assume the superconducting gap to follow the BCS relation $\Delta^{*}=1.764 k_{B} T_{c}$ ). Figure 2 shows that by increasing the bias voltage $V$, spin up and spin down

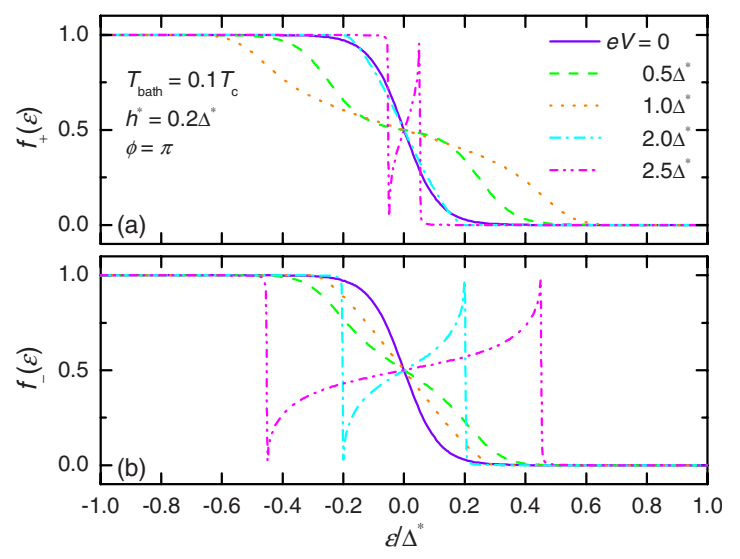

FIG. 3. (Color online) Spin-dependent quasiparticle distribution functions $f_{\sigma}(\varepsilon)$ in the full nonequilibrium limit vs energy $\varepsilon$ for several bias voltages at $\phi=\pi, T_{\text {bath }}=0.1 T_{c}$, and $h^{*}=0.2 \Delta^{*}$. (a) $f_{+}(\varepsilon)$ and (b) $f_{-}(\varepsilon)$.

distributions are shifted in opposite directions on the energy axis, similarly to what is expected in the presence of an effective spin-dependent chemical potential $\left(\mu_{\sigma}^{\text {eff }}\right)$. In particular, $f_{+}(\varepsilon)$ is shifted toward negative energies, while $f_{-}(\varepsilon)$ toward positive energies. Moreover, for $e V \gtrsim \Delta^{*}$, the spindependent chemical potential saturates at $\mu_{\sigma}^{\text {eff }}=-\sigma h^{*}$. As shown in Ref. 30, this effect can be used to electrostatically manipulate the magnetic properties of the $\mathrm{N}$ region. The role of a finite $\Gamma$ (i.e., the presence of quasiparticle states within the gap) can be appreciated in Fig. 2. By increasing $e V$ from 0 to $\Delta^{*}$, the distributions broaden and reflect the heating of the $\mathrm{N}$ region, as discussed in Refs. 22 and 36. This effect is absent for $\Gamma=0$. By further increasing the bias voltage, the distribution functions sharpen due to cooling provided by the superconducting energy gap. ${ }^{22}$

Analogously, in Figs. 3(a) and 3(b), we plot the nonequilibrium distribution functions for spin up and spin down quasiparticles, respectively, for the antiparallel configuration (i.e., $\phi=\pi$ ). Distribution functions are shown vs energy $\varepsilon$ for different values of $V$ and were calculated for the same parameters as in Fig. 2. In this case, up and down distributions remain centered around $\varepsilon=0$ upon biasing (equivalently, their effective chemical potential is always $\left.\mu_{\sigma}^{\text {eff }}=0\right)$, but at a given bias voltage, the features of the distributions are more pronounced for the spin down case. As we shall see in Sec. VI, up and down distributions are characterized by different effective electronic temperatures $\left(T_{\sigma}^{\mathrm{eff}}\right)$. In general, for any angle $\phi$ differing from 0 or $\pi$, the spin-dependent distribution functions $f_{\sigma}(\varepsilon)$ will be characterized by both an effective chemical potential and an effective electronic temperature.

\section{B. Intermediate inelastic scattering}

In the presence of scattering, the approach of Sec. III A cannot be used and one has to resort to the kinetic equation theory. Electrons in metals experience both elastic and inelastic collisions. The latter drive the system to equilibrium and can be expected to hinder the manifestation of the phe- 
nomena discussed in the previous section. At low temperatures (typically below $1 \mathrm{~K}$ ), electron-electron scattering ${ }^{39}$ and scattering with magnetic impurities ${ }^{40,41}$ are the dominant sources of inelastic collisions. ${ }^{3,41,42}$ Since $R_{\mathrm{t}}$ is, in general, large compared to the wire resistance $\left[R_{\mathrm{N}}=t_{\mathrm{N}} /\left(\mathcal{N}_{\mathrm{F}}^{\mathrm{N}} e^{2} D A\right)\right]$, where $\mathcal{N}_{\mathrm{F}}^{\mathrm{N}}$ is the $\mathrm{N}$-region DOS at the Fermi energy and $A$ the wire cross section), we can assume that $f_{\sigma}$ does not depend on the position in the wire. ${ }^{15}$

In the following, we shall analyze the role of inelastic electron-electron relaxation on the quasiparticle distribution. The effect of electron-electron scattering due to the Coulomb interaction on the spin-dependent distributions can be accounted for by solving a pair of coupled stationary kinetic equations,

$$
\begin{aligned}
& D \frac{\partial^{2} f_{+}(\varepsilon)}{\partial x^{2}}=\mathcal{I}_{\text {coll }}^{+}(\varepsilon), \\
& D \frac{\partial^{2} f_{-}(\varepsilon)}{\partial x^{2}}=\mathcal{I}_{\text {coll }}^{-}(\varepsilon),
\end{aligned}
$$

together with the Kuprianov-Lukichev boundary conditions at the NIS interfaces. ${ }^{43}$ In Eq. (5), $\mathcal{I}_{\text {coll }}^{\sigma}(\varepsilon)$ is the net collision rate at energy $\varepsilon$, functional of the distribution functions $f_{\sigma}$, defined by

$$
\mathcal{I}_{\text {coll }}^{\sigma}(\varepsilon)=\mathcal{I}_{\text {coll }}^{\text {in } \sigma}(\varepsilon)-\mathcal{I}_{\text {coll }}^{\text {out } \sigma}(\varepsilon)
$$

where

$$
\begin{aligned}
\mathcal{I}_{\mathrm{coll}}^{\mathrm{in} \sigma}(\varepsilon)= & {\left[1-f_{\sigma}(\varepsilon)\right] \int d \omega \frac{k(\omega)}{2} f_{\sigma}(\varepsilon-\omega) \int d E\left\{f_{+}(E+\omega)\right.} \\
& \left.\times\left[1-f_{+}(E)\right]+f_{-}(E+\omega)\left[1-f_{-}(E)\right]\right\}
\end{aligned}
$$

and

$$
\begin{aligned}
\mathcal{I}_{\text {coll }}^{\text {out } \sigma}(\varepsilon)= & f_{\sigma}(\varepsilon) \int d \omega \frac{k(\omega)}{2}\left[1-f_{\sigma}(\varepsilon-\omega)\right] \int d E\left\{f_{+}(E)\right. \\
& \left.\times\left[1-f_{+}(E+\omega)\right]+f_{-}(E)\left[1-f_{-}(E+\omega)\right]\right\} .
\end{aligned}
$$

In Eqs. (7) and (8), $k(\omega)=\kappa_{\mathrm{ee}} \omega^{-3 / 2}$ according to the theory of the screened Coulomb interaction ${ }^{44}$ for a quasi-onedimensional wire, where $\kappa_{\mathrm{ee}}=\left(\pi \sqrt{2 D} \hbar^{3 / 2} \mathcal{N}_{\mathrm{F}}^{\mathrm{N}} A\right)^{-1} \cdot{ }^{45,46}$ By rewriting Eq. (5) in dimensionless units, ${ }^{15}$ the strength of the electron-electron interaction can be expressed as $\mathcal{K}_{\text {coll }}$ $=\left(R_{\mathrm{t}} / R_{\mathrm{N}}\right)\left(t_{\mathrm{N}}^{2} \kappa_{\mathrm{ee}} / D\right) \sqrt{\Delta}=\left(t_{\mathrm{N}} / \sqrt{2}\right)\left(R_{\mathrm{t}} / R_{K}\right) \sqrt{\Delta / \hbar D}$, where $R_{K}$ $=h / 2 e^{2}$. We note that the strength of the electron-electron interaction turns out to be proportional to the length of the wire as well as to the tunnel barrier resistance.

We solved Eq. (5) with $h^{*}=0.2 \Delta^{*}, e V=\Delta^{*}$, and $T$ $=0.1 T_{c}$ for several $\mathcal{K}_{\text {coll }}$ values. ${ }^{44,45}$ The effect of electronelectron scattering on the quasiparticle distribution functions is displayed in Figs. 4 and 5 for $\phi=0$ and $\phi=\pi$, respectively. For the $\phi=0$ case, by increasing Coulomb interactions, the quasiparticle distributions are forced toward thermal ones still characterized by different chemical potentials for both spin species. ${ }^{30}$ In the antiparallel configuration (see Fig. 5), the effect of inelastic relaxation is similar, but now the spindependent distribution function will coincide for sufficiently large $\mathcal{K}_{\text {coll }}$ values. It is easy to recognize that, in both cases,

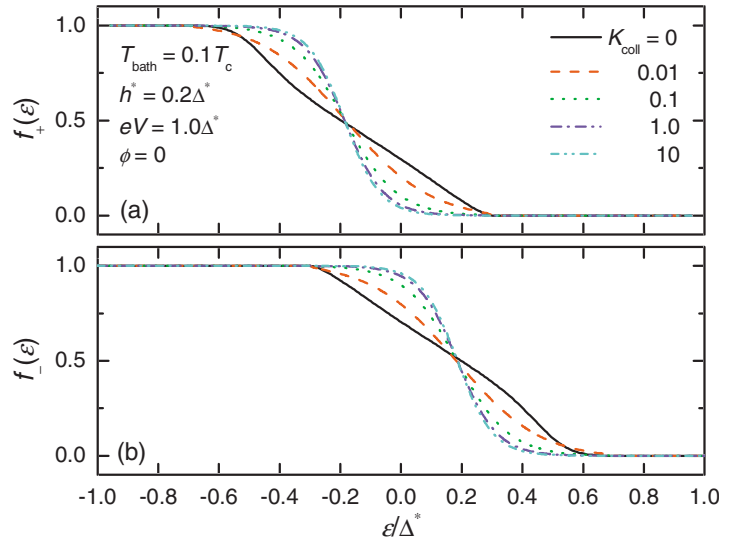

FIG. 4. (Color online) Spin-dependent quasiparticle distribution functions $f_{\sigma}(\varepsilon)$ vs energy $\varepsilon$ calculated for several $\mathcal{K}_{\text {coll }}$ values at $\phi=0, e V=\Delta^{*}, T_{\text {bath }}=0.1 T_{c}$, and $h^{*}=0.2 \Delta^{*}$. (a) $f_{+}(\varepsilon)$ and (b) $f_{-}(\varepsilon)$.

a thermal Fermi-like distribution is reached for $\mathcal{K}_{\text {coll }}$ of the order of 10. Assuming parameters for a realistic $\mathrm{Al} / \mathrm{Al}_{2} \mathrm{O}_{3} / \mathrm{Ag}$ SINIS microstructure ${ }^{21,36}$ (with $\Delta \simeq 200 \mu \mathrm{eV}$, $D=0.02 \mathrm{~m}^{2} \mathrm{~s}^{-1}$, and $\left.R_{\mathrm{t}}=1 \mathrm{k} \Omega\right), \mathcal{K}_{\text {coll }}=10$ corresponds to a rather long $\mathrm{N}$ region, $t_{\mathrm{N}} \approx 47 \mu \mathrm{m}$.

\section{Strong inelastic scattering: Quasiequilibrium limit}

This is the regime characterized by the fact that the electron-electron interaction is so strong that quasiparticles can reach an equilibrium (Fermi-like) distribution, while the electron-phonon coupling is negligible. ${ }^{22}$ Such distributions are characterized by the quasiequilibrium chemical potential and temperature. Since electron-electron interaction occurs between quasiparticles irrespective of their spin (in the absence of spin-mixing mechanisms), the quasiequilibrium temperature $\left(T^{\mathrm{qe}}\right)$ will be independent of spin and different from the temperature of the phonon bath $T_{\text {bath }}$. On the contrary, since electron-electron interaction redistributes the energy among electrons of a given spin species, in the absence of spin-mixing mechanisms, the quasiequilibrium chemical potential $\left(\mu_{\sigma}^{\mathrm{qe}}\right)$ will depend on the spin. This is a conse-

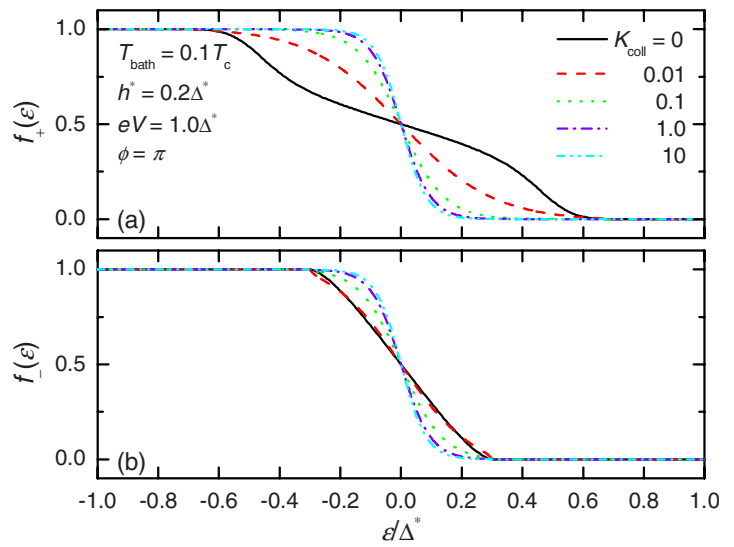

FIG. 5. (Color online) Spin-dependent quasiparticle distribution functions $f_{\sigma}(\varepsilon)$ vs energy $\varepsilon$ calculated for several $\mathcal{K}_{\text {coll }}$ values at $\phi=\pi, e V=\Delta^{*}, T_{\text {bath }}=0.1 T_{c}$, and $h^{*}=0.2 \Delta^{*}$. (a) $f_{+}(\varepsilon)$ and (b) $f_{-}(\varepsilon)$. 
quence of the fact that the number of electrons of a given spin must be conserved. Furthermore, both quasiequilibrium chemical potential and quasiequilibrium temperature will depend on $\phi$; therefore, they will be different for parallel and antiparallel configurations.

In the absence of spin-flip mechanisms, the quasiequilibrium distribution functions can be calculated by imposing the conservation of particle currents, independently for the two spin species, together with a balance equation for the heat currents. In particular, in the former case, we require that

$$
I_{\sigma}^{\mathrm{L}}\left(V, h^{*}, \phi\right)=I_{\sigma}^{\mathrm{R}}\left(V, h^{*}, \phi\right),
$$

where

$$
I_{\sigma}^{\mathrm{L}}\left(V, h^{*}, \phi\right)=\frac{1}{e R_{\mathrm{t}}} \int d \varepsilon \mathcal{N}_{\sigma}^{\mathrm{S}_{1}}(\varepsilon)\left[\mathcal{F}^{\mathrm{S}_{1}}(\varepsilon)-f_{\sigma}(\varepsilon)\right]
$$

and

$$
\begin{aligned}
I_{\sigma}^{\mathrm{R}}\left(V, h^{*}, \phi\right)= & \frac{1}{e R_{\mathrm{t}}} \int d \varepsilon\left[\mathcal{N}_{\sigma}^{\mathrm{S}_{2}}(\varepsilon) a(\phi)+\mathcal{N}_{-\sigma}^{\mathrm{S}_{2}}(\varepsilon) b(\phi)\right]\left[f_{\sigma}(\varepsilon)\right. \\
& \left.-\mathcal{F}^{\mathrm{S}_{2}}(\varepsilon)\right]
\end{aligned}
$$

are the electric currents flowing through the left (L) and right (R) NIS interface. Note that in contrast to the full nonequilibrium regime where the tunneling rates are set to be equal at each energy, here the conservation involves the total currents since the electron-electron interaction mixes the energy of the electrons. In the absence of electron-phonon coupling, the only contribution to the heat flux is the heat current flowing off the $\mathrm{N}$ region through each NIS interface. The latter is given by

$$
J_{\sigma}^{\mathrm{L}}\left(V, h^{*}, \phi\right)=\frac{1}{e^{2} R_{\mathrm{t}}} \int d \varepsilon \varepsilon \mathcal{N}_{\sigma}^{\mathrm{S}_{1}}(\varepsilon)\left[f_{\sigma}(\varepsilon)-\mathcal{F}^{\mathrm{S}}(\varepsilon)\right],
$$

for the left NIS contact, and by

$$
\begin{aligned}
J_{\sigma}^{\mathrm{R}}\left(V, h^{*}, \phi\right)= & \frac{1}{e^{2} R_{\mathrm{t}}} \int d \varepsilon \varepsilon\left[\mathcal{N}_{\sigma}^{\mathrm{S}_{2}}(\varepsilon) a(\phi)+\mathcal{N}_{-\sigma}^{\mathrm{S}_{2}}(\varepsilon) b(\phi)\right] \\
& \times\left[f_{\sigma}(\varepsilon)-\mathcal{F}^{\mathrm{S}_{2}}(\varepsilon)\right],
\end{aligned}
$$

for the right contact. The balance equation for the heat flux thus simply reads

$$
\sum_{\sigma}\left[J_{\sigma}^{\mathrm{L}}\left(V, h^{*}, \phi\right)+J_{\sigma}^{\mathrm{R}}\left(V, h^{*}, \phi\right)\right]=0 .
$$

By assuming that $f_{\sigma}=f_{0}\left(\varepsilon-\mu_{\sigma}^{\mathrm{qe}}, T^{\mathrm{qe}}\right)$ and solving Eqs. (9) and (14), the temperature and chemical potentials can be easily determined. It turns out that while in the antiparallel alignment spin up and down distributions are equal, in the parallel one, the two spin components have equal effective electronic temperature (though different from the antiparallel alignment) but opposite effective chemical potential (see Figs. 4 and 5 for large $\mathcal{K}_{\text {coll }}$ values). Although the quasiequilibrium regime might seem an unrealistic limit, it actually describes the case of a strong electron-electron interaction quite well. Indeed, according to our calculations (Sec. III B), quasiequilibrium distributions are already reached for an electron-electron collision strength $\mathcal{K}_{\text {coll }} \simeq 10$. In the follow- ing sections, we shall investigate the impact of quasiequilibrium on spin-dependent transport properties.

\section{ELECTRIC CURRENT}

The transport properties of the FS-I-N-SF structure are determined by the spin-dependent distribution functions $f_{\sigma}$. We note that although a Josephson current can flow through the system, its theoretical description is beyond the scope of the present paper. As a matter of fact, we shall be only concerned with the quasiparticle transport. Furthermore, although similar results for tunnel magnetoresistance and current polarization could be obtained in a FS-I-SF structure (i.e., without the $\mathrm{N}$ interlayer) and not relying on nonequilibrium, the present system possesses a crucial advantage. In fact, a FS-I-SF structure implies an additional undesired Josephson current, which can be fairly large as compared to the quasiparticle current (around 1 order of magnitude larger than the quasiparticle current relevant for high tunnel magnetoresistance and current polarization, see, for example, Ref. 33). Such supercurrent could be suppressed, for instance, by the application of an additional in-plane magnetic field. This field, however, would largely exceed that required to control the orientation of $h^{*}$. By contrast, in the FS-I-NI-SF system, the supercurrent can be kept extremely small up to a large extent, depending mainly on $t_{\mathrm{N}}$, on the tunnel barrier transmissivity, and on the $\mathrm{N}$-interlayer material parameters. A simple estimate for the Josephson coupling in our structure reveals that the supercurrent can be from 1 to several orders of magnitude smaller than the quasiparticle current (see, for example, Ref. 47).

The quasiparticle current $I$ (e.g., evaluated at the left interface) is given by

$$
I\left(V, h^{*}, \phi\right)=\sum_{\sigma} I_{\sigma}^{\mathrm{L}}\left(V, h^{*}, \phi\right) .
$$

Figure 6(a) displays the electric current in full nonequilibrium vs bias voltage $V$ calculated for several angles $\phi$ at $h^{*}=0.2 \Delta^{*}$ and $T=0.1 T_{c}$. A sizable current starts to flow only when the voltage $V$ is such that the DOS is finite for both superconductors in some range of energies. For $\phi=0$, the current rises sharply at $|e V|=2 \Delta^{*}$, similarly to the quasiparticle current of a SIS junction (also in the presence of an in-plane magnetic field ${ }^{35}$ ). In this case, in fact, the DOS of a given spin is shifted by the Zeeman energy in the same direction for both superconductors. In contrast, for $\phi=\pi$, current sets off at $|e V|=2\left(\Delta^{*}-h^{*}\right)$.

Figure 6(b) shows the nonequilibrium differential conductance

$$
G\left(V, h^{*}, \phi\right)=\frac{d I\left(V, h^{*}, \phi\right)}{d V},
$$

calculated for the same values as in Fig. 6(a). Additional features are present at $|e V|=2 h^{*}$, which are strongly temperature dependent and vanish in the limit $T \rightarrow 0$ (the zero-bias conductance peak for $\phi \neq \pi$ resembles that typical of a SIS junction composed of identical superconductors ${ }^{48}$ ). These are a consequence of the overlapping of the superconducting 

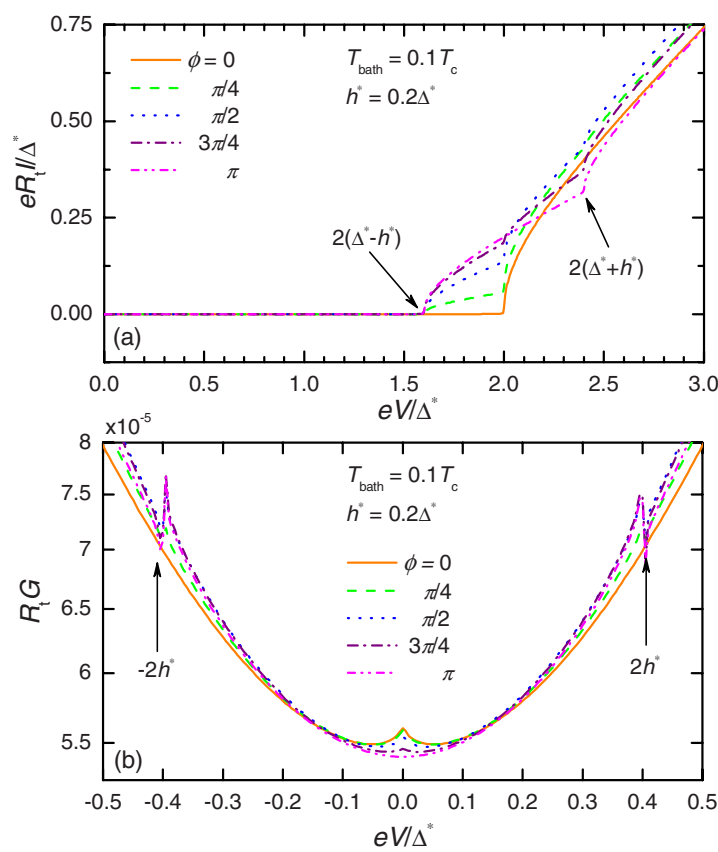

FIG. 6. (Color online) (a) Nonequilibrium current vs bias voltage $V$ for several angles $\phi$ at $T_{\text {bath }}=0.1 T_{c}$ and $h^{*}=0.2 \Delta^{*}$. (b) Nonequilibrium differential conductance $G$ vs $V$ calculated for the same values as in (a).

DOSs where only thermally activated quasiparticles exist at finite temperature.

All these simply reflects how the spin-dependent DOS in each superconductor contributes to the total quasiparticle current at different $V$. This can be easily visualized by inspecting Fig. 7, which shows idealized finite-temperature exchange field-split superconducting DOS for parallel spin species, at different bias voltages $V$ and for the case $\phi=\pi$. In this case, the DOS of $\mathrm{S}_{1}$ is shifted in the opposite direction with respect to that of $S_{2}$ [see Fig. 7(a) for $V=0$ ]. Then, by biasing the structure, the required voltage for a current to flow is smaller with respect to the $\phi=0$ case, i.e., $e V$ $=2\left(\Delta^{*}-h^{*}\right)[$ see Fig. $7(\mathrm{~b})]$. In the same way, for negative voltages, the current sets off at $e V=-2\left(\Delta^{*}+h^{*}\right)$, as shown in Fig. 7(d). It is also clear that antiparallel spin species will give rise to features at the opposite bias voltage, therefore explaining the origin of additional feature appearing at $\mathrm{eV}$ $=2\left(\Delta^{*}+h^{*}\right)$. For intermediate values of $\phi$, features are present at $|e V|=2\left(\Delta^{*} \pm h^{*}\right)$ and at $|e V|=2 \Delta^{*}$ since contributions from both $\phi=0$ and $\phi=\pi$ configurations are present. Of particular relevance is the voltage interval $2\left(\Delta^{*}-h^{*}\right) \leqslant|e V|$ $\leqslant 2 \Delta^{*}$. By increasing $\phi$ from 0 to $\pi$, the current is enhanced from a vanishingly small value up to a finite value leading to a spin-valve effect.

It is noteworthy to mention that the nonequilibrium condition is essential for the observation of the spin-valve effect. At equilibrium, the distribution functions in the $\mathrm{N}$ layer would be thermal and spin independent.

\section{MAGNETORESISTANCE}

The spin-valve properties of the FS-I-N-I-SF setup can be evaluated quantitatively by analyzing the tunnel magnetoresistance ratio (TMR), defined as
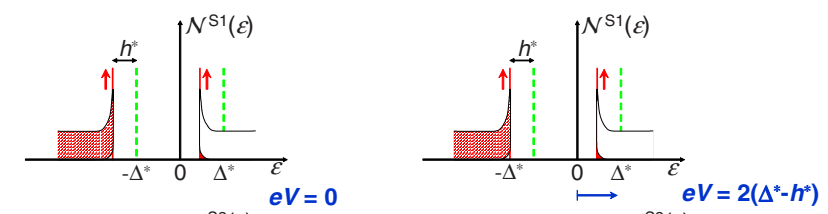

(a)

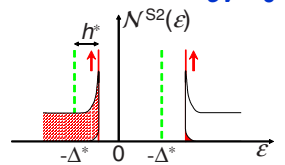

(b)

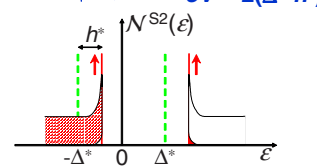

(c)

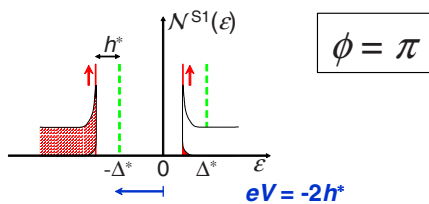

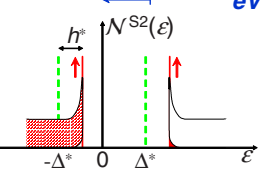

(d)

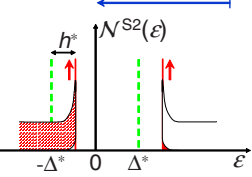

FIG. 7. (Color online) Idealized finite-temperature exchange field-split density of states $\mathcal{N}^{\mathrm{S}_{1,2}}$ of $\mathrm{S}_{1,2}$ for parallel spin species, at $\phi=\pi$ and different bias voltages $V$. In particular, (b), (c), and (d) show how features in the tunneling current originate at $e V=2\left(\Delta^{*}\right.$ $\left.-h^{*}\right), e V=-2 h^{*}$, and $e V=-2\left(\Delta^{*}+h^{*}\right)$, respectively. Antiparallel spin species gives rise to features at opposite voltages. Greendashed lines represent the superconducting DOS in the absence of the exchange field.

$$
\operatorname{TMR}\left(V, h^{*}, \phi\right)=\frac{G\left(V, h^{*}, \phi\right)-G\left(V, h^{*}, 0\right)}{G\left(V, h^{*}, 0\right)}
$$

Figure 8(a) displays the absolute value of the nonequilibrium

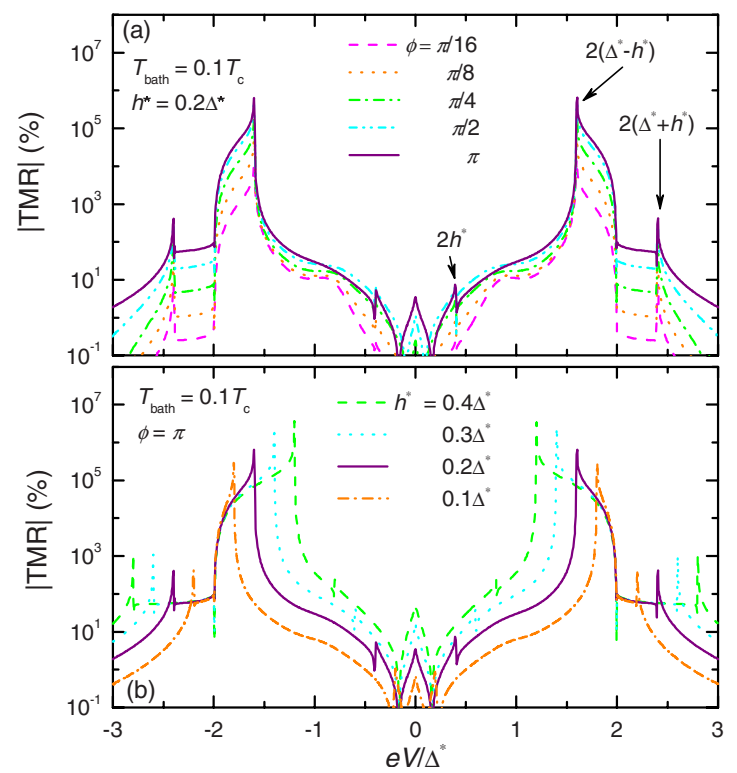

FIG. 8. (Color online) (a) Nonequilibrium tunnel magnetoresistance ratio $|\mathrm{TMR}|$ vs $V$ calculated for several angles $\phi$ at $T_{\text {bath }}$ $=0.1 T_{c}$ and $h^{*}=0.2 \Delta^{*}$. (b) $|\mathrm{TMR}|$ vs $V$ for different $h^{*}$ values at $T=0.1 T_{c}$ and $\phi=\pi$. 


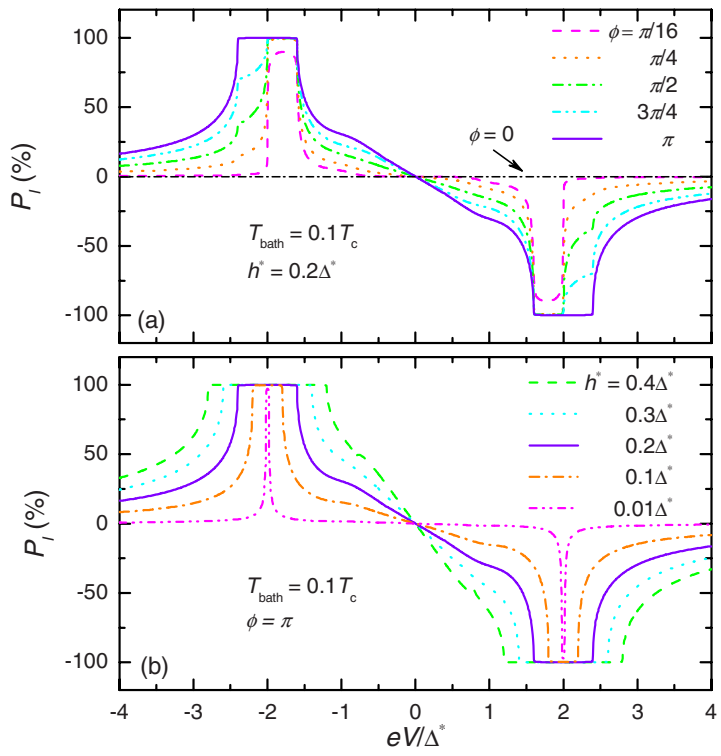

FIG. 9. (Color online) (a) Nonequilibrium current polarization $P_{I}$ vs $V$ calculated for several angles $\phi$ at $T_{\text {bath }}=0.1 T_{c}$ and $h^{*}$ $=0.2 \Delta^{*}$. (b) $P_{I}$ vs $V$ for different $h^{*}$ values at $T_{\text {bath }}=0.1 T_{c}$ and $\phi$ $=\pi$.

TMR vs bias voltage $V$ calculated for several angles $\phi$ at $T$ $=0.1 T_{c}$ and $h^{*}=0.2 \Delta^{*}$. For $2\left(\Delta^{*}-h^{*}\right) \leqslant|e V| \leqslant 2 \Delta^{*}$, the TMR increases monotonically by increasing $\phi$ and is maximized at $\phi=\pi$ where it reaches huge values exceeding $10^{6} \%$. We note that in the limit $T=0$ and $\Gamma=0,|\mathrm{TMR}|$ diverges, realizing an ideal full spin-valve effect. The nonequilibrium TMR behavior for several exchange field values is shown in Fig. 8(b), at $T=0.1 T_{c}$ and $\phi=\pi$. By decreasing $h^{*}$, the maximum TMR value reduces and so does the voltage interval of larger magnetoresistance. Larger $h^{*}$ values are thus preferable in order to extend the voltage window for optimized operation and to maximize the TMR.

The spin-filtering properties of this system can be quantified by inspecting the current polarization $\left(P_{I}\right)$, defined as

$$
P_{I}\left(V, h^{*}, \phi\right)=\frac{I_{+}^{\mathrm{L}}\left(V, h^{*}, \phi\right)-I_{-}^{\mathrm{L}}\left(V, h^{*}, \phi\right)}{I_{+}^{\mathrm{L}}\left(V, h^{*}, \phi\right)+I_{-}^{\mathrm{L}}\left(V, h^{*}, \phi\right)} .
$$

The calculated nonequilibrium $P_{I}$ vs $V$ is displayed in Fig. 9(a) for several $\phi$ values, at $T=0.1 T_{c}$ and $h^{*}=0.2 \Delta^{*}$. Upon increasing $\phi$, two intervals of $100 \%$ spin-polarized current develop for $2\left(\Delta^{*}-h^{*}\right) \leqslant|e V| \leqslant 2 \Delta^{*}$, extending to wider regions $\left[2\left(\Delta^{*}-h^{*}\right) \leqslant|e V| \leqslant 2\left(\Delta^{*}+h^{*}\right)\right]$ as $\phi$ approaches $\pi$. For $\phi=0, P_{I}$ vanishes like in SIS junctions with an in-plane magnetic field. ${ }^{35}$ Depending on bias, fully spin-polarized currents of both parallel and antiparallel spin species can be obtained. The structure can thus also be operated as a controllable spin filter by changing the orientation of $\boldsymbol{h}_{2}$ as well as by varying $V$. Figure 9(b) shows $P_{I}$ vs $V$ for several $h^{*}$ at $T=0.1 T_{c}$ and $\phi=\pi$. The net effect of increasing $h^{*}$ is to widen the regions of $100 \%$ spin-polarized current.

It is important to discuss the effect of the smearing parameter $\Gamma$ (which controls the presence of quasiparticle states within the superconducting gap) on the magnetoresistance

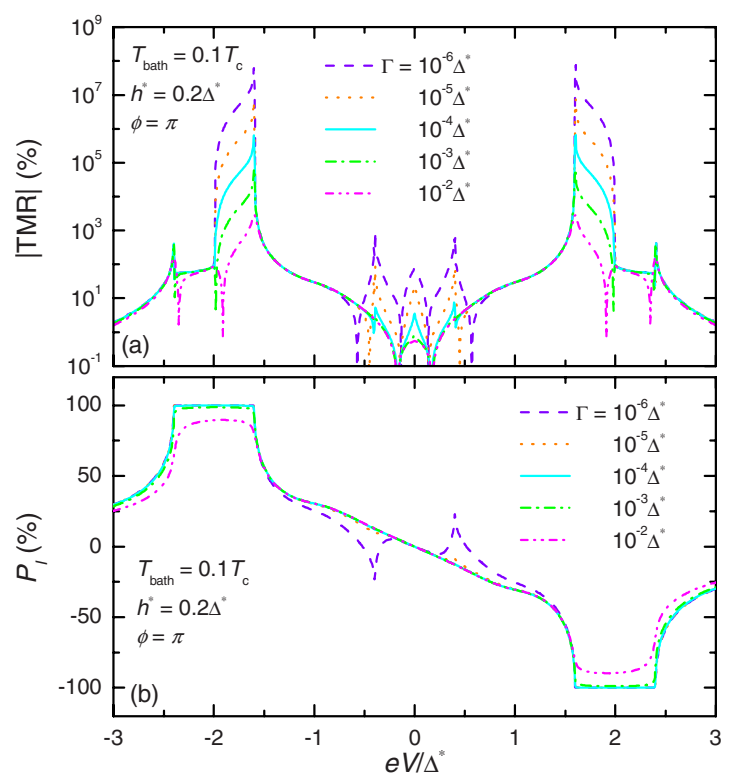

FIG. 10. (Color online) (a) Nonequilibrium tunnel magnetoresistance ratio $|\mathrm{TMR}|$ vs $V$ calculated for several $\Gamma$ values at $T_{\text {bath }}$ $=0.1 T_{c}, h^{*}=0.2 \Delta^{*}$, and $\phi=\pi$. (b) Nonequilibrium $P_{I}$ vs $V$ calculated for the same values as in (a).

and current polarizations. As shown in Fig. 10(a), by increasing $\Gamma$, the TMR value decreases mostly in the region $2\left(\Delta^{*}\right.$ $\left.-h^{*}\right) \leqslant|e V| \leqslant 2 \Delta^{*}$, while for other values of $V$, almost no changes are found apart from some smoothing of sharp features. In particular, the normal character of transport is strengthened by increasing $\Gamma$, which causes a suppression of the large TMR value. The latter, indeed, is a consequence of the presence of the superconducting gap. On the contrary, the impact of $\Gamma$ on $P_{I}$, plotted in Fig. 10(b) as a function of the voltage $V$, is much weaker: the polarization in the range $2\left(\Delta^{*}-h^{*}\right) \leqslant|e V| \leqslant 2\left(\Delta^{*}+h^{*}\right)$ is almost insensitive to $\Gamma$, being slightly reduced only for $\Gamma$ values as large as $10^{-2} \Delta^{*}$.

TMR values are expected to be marginally affected by the presence of electron-electron relaxation in the $\mathrm{N}$ layer. Indeed, as discussed in Secs. III B and III C, Coulomb interaction allows quasiparticles to exchange energy (through inelastic collisions) without coupling the two spin species. As shown in Figs. 4 and 5, inelastic scattering leaves the two distributions $f_{+}(\varepsilon)$ and $f_{-}(\varepsilon)$ strongly spin dependent in the parallel configuration, while making them to coincide in the antiparallel configuration, so that both magnetoresistance and current polarizations are expected to be only slightly affected. Indeed, TMR is only marginally affected even in the quasiequilibrium regime, as shown in Fig. 11(a), where we compare TMR at $\phi=\pi$, as a function of $V$, for the full nonequilibrium and the quasiequilibrium regimes. The effect of energy redistribution characteristic of quasiequilibrium consists merely in a smoothing of some of the sharp features present in the nonequilibrium limit. In Fig. 11(b), we compare the plots of $P_{I}$ at $\phi=\pi$ as functions of $V$ for both regimes. In particular, quasiequilibrium displays a reduction of polarization for $|V|>2\left(\Delta^{*}-h^{*}\right)$ and an increase of polarization for $|V|<2\left(\Delta^{*}-h^{*}\right)$. Nevertheless, $P_{I}$ values as large as $100 \%$ can be obtained in the quasiequilibrium limit as well. 

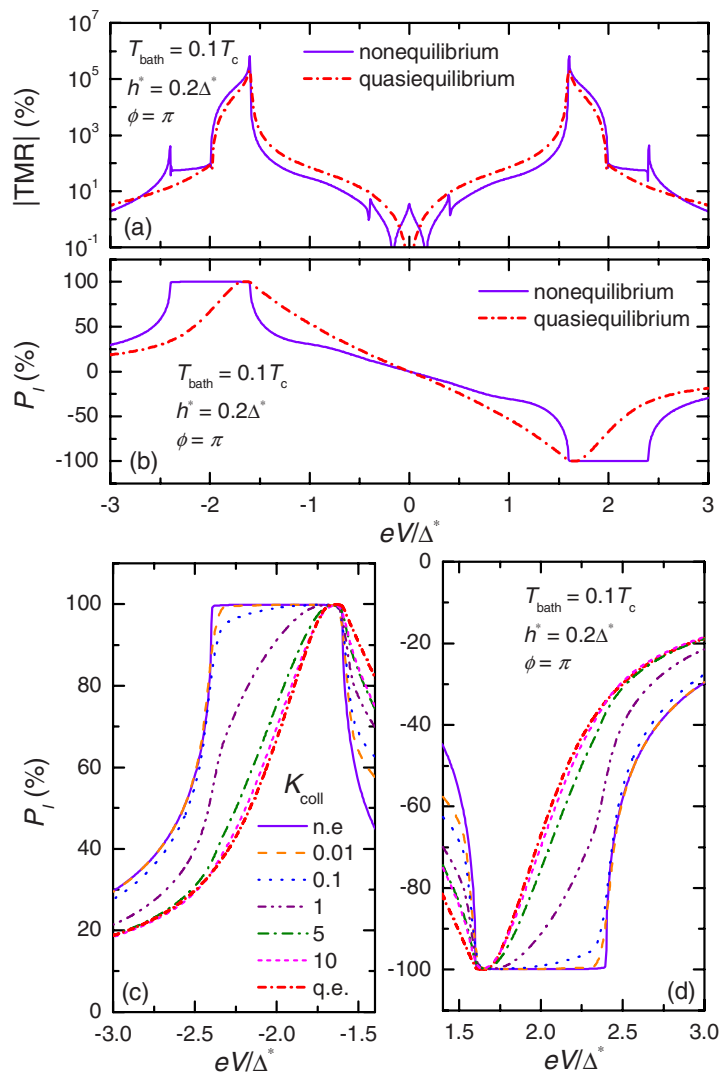

FIG. 11. (Color online) (a) Tunnel magnetoresistance ratio |TMR | vs $V$ in nonequilibrium (solid line) and quasiequilibrium (dash-dotted line) at $T_{\text {bath }}=0.1 T_{c}, h^{*}=0.2 \Delta^{*}$, and $\phi=\pi$. (b) Current polarization $P_{I}$ vs $V$ in nonequilibrium (solid line) and quasiequilibrium (dash-dotted line) calculated for the same values as in (a). [(c) and (d)] $P_{I}$ vs $V$ in the intervals of large polarization calculated for several $\mathcal{K}_{\text {coll }}$ values and the same parameters as in (a).

The full evolution of $P_{I}$ as a function of $\mathcal{K}_{\text {coll }}$ in the relevant intervals of large polarization is shown in Figs. 11(c) and $11(\mathrm{~d})$. It is easy to notice the gradual smearing of $P_{I}$ by increasing the electron-electron interaction strength, and that the quasiequilibrium limit is already reached for $\mathcal{K}_{\text {coll }} \sim 10$ (see also the discussion at the end of Sec. III B). However, in FS-I-N-I-SF realistic structures, it should be possible to keep $\mathcal{K}_{\text {coll }} \sim 0.1$ or smaller so that current polarization would be somewhat similar to that obtained in the full nonequilibrium limit.

By contrast, TMR must decrease if spin-flip processes mix the spin-dependent distributions. In metals and at low temperature (typically below $\sim 1 \mathrm{~K}$ ), such processes are normally caused by the presence of magnetic impurities in the $\mathrm{N}$ layer. Spin-flip scattering can be suppressed by limiting the magnetic-impurity content in the $\mathrm{N}$ layer and by choosing $t_{\mathrm{N}} \ll \lambda_{\text {sf }}$ [the spin-flip relaxation length $\lambda_{\text {sf }}$ is of the order of some micrometers in metals such as $\mathrm{Cu}$ or Au (Refs. 49 and 50)]. These constraints can be met fairly easily experimentally in multilayered or planar structures like the ones presented here.

\section{SPIN-DEPENDENT EFFECTIVE TEMPERATURE}

Even in the nonequilibrium case, it is interesting to characterize the distribution function through an effective temperature and an effective chemical potential. Such effective quantities can be meaningfully defined by fictitiously connecting the $\mathrm{N}$ region to a large normal metal reservoir through an insulating barrier that is sufficiently transparent to allow the flow of quasiparticles but opaque enough not to alter the nonequilibrium condition of the electrons. One can identify the effective chemical potential $\left(\mu_{\sigma}^{\text {eff }}\right)$ of the out-ofequilibrium electron gas in the $\mathrm{N}$ region with the chemical potential the reservoir must possess in order for the particle current to be zero. The effective temperature $\left(T_{\sigma}^{\mathrm{eff}}\right)$, on the other hand, is taken to be equal to the one the reservoir must have in order for the heat current to be zero. ${ }^{51}$ These conditions can be expressed by the following two equations:

$$
\begin{gathered}
\int d \varepsilon\left[f_{\sigma}(\varepsilon)-f_{0}\left(\varepsilon-\mu_{\sigma}^{\mathrm{eff}}, T_{\sigma}^{\mathrm{eff}}\right)\right]=0 \\
\int d \varepsilon \varepsilon\left[f_{\sigma}(\varepsilon)-f_{0}\left(\varepsilon-\mu_{\sigma}^{\mathrm{eff}}, T_{\sigma}^{\mathrm{eff}}\right)\right]=0
\end{gathered}
$$

where we have assumed that the DOS of the $\mathrm{N}$ reservoir is equal to that of the $\mathrm{N}$ layer. We wish to warn the reader that the words "temperature" and "chemical potential" have to be taken in a loose sense, especially when the distributions are very different from equilibrium functions. They are merely two parameters which grasp important characteristic properties of the distributions, related, namely, to particle and heat transport.

A general expression for the effective temperature can be easily derived through the Sommerfeld expansion, ${ }^{36}$ obtaining

$$
T_{\sigma}^{\mathrm{eff}}=\frac{\sqrt{6}}{\pi k_{B}} \sqrt{\int_{-\infty}^{\infty} d \varepsilon \varepsilon\left[f_{\sigma}(\varepsilon)-f_{0}\left(\varepsilon-\mu_{\sigma}^{\mathrm{eff}}, T=0\right)\right]},
$$

where

$$
\mu_{\sigma}^{\mathrm{eff}}=\int_{-\infty}^{\infty} d \varepsilon\left[f_{\sigma}(\varepsilon)-\theta(\varepsilon)\right]
$$

and $\theta(\varepsilon)$ is the Heaviside step function. Equation (21) yields the true spin-dependent electron temperature in (quasi)equilibrium. Furthermore, in the present FS-I-N-I-SF system, $T_{\sigma}^{\mathrm{eff}}$ turns out to depend on the strength of electron-electron interaction (i.e., on $\mathcal{K}_{\text {coll }}$ ) as we shall show in the following, due to heat exchange with FS reservoirs with nonconstant DOS. ${ }^{22,36}$

In Figs. 12(a) and 12(b), we plot the calculated effective temperatures in the full nonequilibrium limit (i.e., for $\mathcal{K}_{\text {coll }}$ $=0)$, normalized with respect to the bath temperature $\left(T_{\text {bath }}\right.$ $\left.=0.1 T_{c}\right)$, as a function of the applied bias voltage $V$ for $\phi$ $=0$ and $\phi=\pi$, respectively. In the former case, there is no spin dependence and $T_{ \pm}^{\mathrm{eff}}$ is an even function of the bias voltage. Starting from the equilibrium condition (i.e., at $V$ $=0$ where the $\left.T_{ \pm}^{\text {eff }}=T_{\text {bath }}\right)$, the temperature first increases reaching a maximum around $e V / \Delta^{*}=0.8$ and thereafter de- 


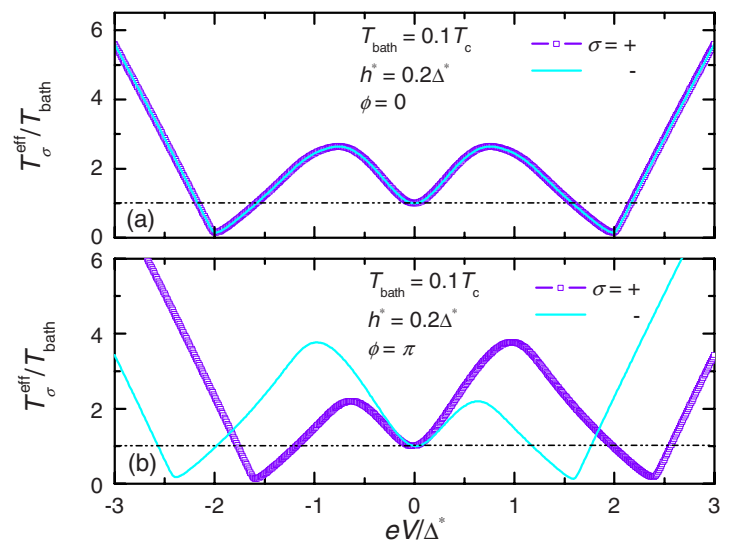

FIG. 12. (Color online) (a) Nonequilibrium spin-dependent electron effective temperature $T_{\sigma}^{\text {eff }}$ vs $V$ calculated for $\phi=0$ at $T_{\text {bath }}$ $=0.1 T_{c}$ and $h^{*}=0.2 \Delta^{*}$. (b) $T_{\sigma}^{\text {eff }}$ vs $V$ calculated for $\phi=\pi$ at $T_{\text {bath }}$ $=0.1 T_{c}$ and $h^{*}=0.2 \Delta^{*}$.

creases down to the minimum around $e V / \Delta^{*}=2$. The initial increase is the "anomalous heating" due to the presence of a finite DOS within the superconducting gap, ${ }^{22,36}$ while the minimum reflects the usual electron "cooling," which is maximized for voltages around twice the value of the gap ${ }^{22}$ (see the discussion in Sec. III A). Such features are present also in the absence of an exchange field, with negligible quantitative differences. By contrast, the effect of the exchange field is manifested in the antiparallel configuration. Indeed, the effective temperatures are different for the two spin species, though related according to the relation $T_{\sigma}^{\text {eff }}(-V)=T_{-\sigma}^{\text {eff }}(V)$. In particular, minima are shifted by an amount equal to $2 h^{*}$ toward higher (lower) voltages for spin up (down) electrons. This fact can also be understood through the schemes shown in Fig. 7, as due to the shift, introduced by the exchange field, of the DOS of the two superconductors in opposite directions. For spin up electrons, maximum cooling occurs for $e V=2\left(\Delta^{*}-h^{*}\right)$ [see Fig. 7(b)] and for $e V=-2\left(\Delta^{*}+h^{*}\right)$ [see Fig. 7(d)]. For spin down electrons, maximum cooling occurs at opposite voltages. The position and amplitude of maxima turns out to be a function of the exchange field, as well as of the smearing parameter $\Gamma$. Notably, as shown in Fig. 12(b), the spin-dependent effective temperatures can be largely different upon voltage biasing the structure.

In Fig. 13(a), we plot the nonequilibrium effective temperature difference $\delta T^{\mathrm{eff}}=T_{+}^{\mathrm{eff}}-T_{-}^{\mathrm{eff}}$ normalized to the bath temperature $\left(T_{\text {bath }}=0.1 T_{c}\right)$ versus $V$ at $\phi=\pi$ and for different values of the exchange field $h^{*} . \delta T^{\mathrm{eff}}$ is odd in the applied voltage and is more pronounced for larger values of $h^{*}$. For positive values of $V$, the maximum (as large as 500\% at this bath temperature) corresponds to the anomalous heating (occurring around $e V / \Delta^{*} \simeq 1$ ), while the minimum occurs for $e V / \Delta^{*} \gtrsim 2$ and moves to higher values as $h^{*}$ increases.

The effect of electron-electron inelastic collisions is addressed in Fig. 13(b), which shows $\delta T^{\mathrm{eff}} / T_{\text {bath }}$ at $\phi=\pi$ as a function of the collision strength $\mathcal{K}_{\text {coll }}$ for $e V=1.0 \Delta^{*}, h^{*}$ $=0.2 \Delta^{*}$, and $T_{\text {bath }}=0.1 T_{c}$. We find a dramatic effect of electron-electron interaction that leads to a strong suppression of the effective temperature difference on the scale of

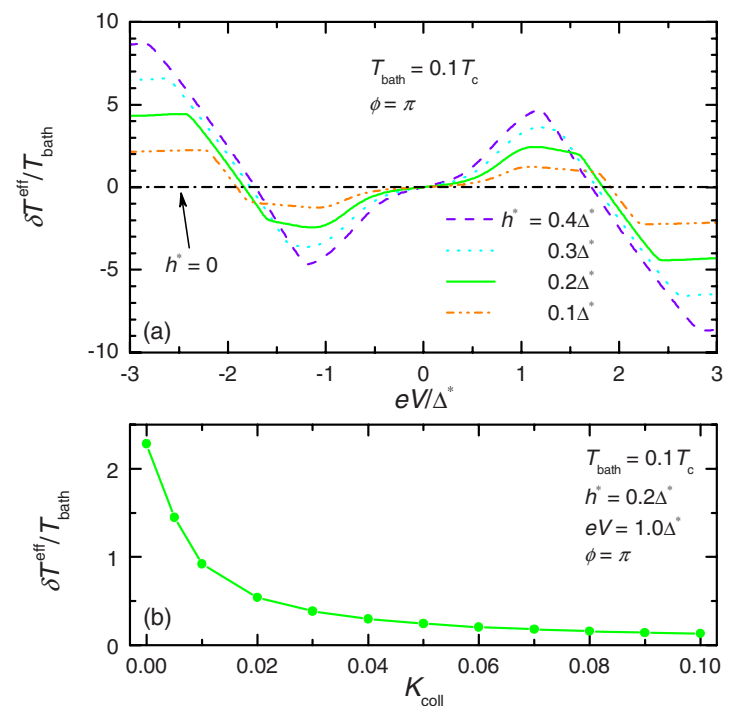

FIG. 13. (Color online) (a) Difference of the nonequilibrium spin-dependent electron effective temperatures $\delta T^{\text {eff }}$ vs $V$ calculated for different $h^{*}$ values at $T_{\text {bath }}=0.1 T_{c}$ and $\phi=\pi$. (b) $\delta T^{\text {eff }}$ vs $\mathcal{K}_{\text {coll }}$ calculated at $e V=1.0 \Delta^{*}$ for $T_{\text {bath }}=0.1 T_{c}, h^{*}=0.2 \Delta^{*}$, and $\phi=\pi$.

$\mathcal{K}_{\text {coll }} \simeq 0.1$. With the material parameters given in Sec. III B, this would correspond to a $\mathrm{N}$ region with $t_{\mathrm{N}} \approx 470 \mathrm{~nm}$.

The possibility to have access to different spin-dependent electronic temperatures suggests that we investigate the potential for the implementation of spintronic devices. In particular, we conceive a nanostructure like that shown in Fig. 14(a), where an additional voltage-biased superconducting electrode $\left(\mathrm{S}_{3}\right)$ is tunnel coupled to the $\mathrm{N}$ region through a junction of resistance $R_{\mathrm{p}} \gg R_{\mathrm{t}},{ }^{52}$ while the exchange fields in $\mathrm{S}_{1,2}$ are arranged in the antiparallel configuration $(\phi=\pi)$. The presence of a superconducting extraction lead is crucial since in the tunneling process, the quasiparticle current through $\mathrm{S}_{3}$ will depend exponentially on the electron temperature in $\mathrm{N}^{22}$ On one hand, the setup considered allows direct measurement of the spin-dependent electron temperatures and $\mathrm{S}_{3}$ may act as a thermometer. ${ }^{22} \mathrm{~S}_{3}$ provides, in fact, access to the whole distribution functions $f_{\sigma}(\varepsilon)$ from the voltage-dependent differential conductance of the $\mathrm{NIS}_{3}$ junction. ${ }^{3}$ On the other hand, upon biasing the $S_{3}$ electrode with $V_{\text {bias }}$, the existence of different spin-dependent temperatures in the normal metal region yields a finite current polarization $P_{I}$ defined in the usual way as

$$
P_{I}\left(V, V_{\text {bias }}, h^{*}\right)=\frac{I_{+}^{\mathrm{S}_{3}}\left(V, V_{\mathrm{bias}}, h^{*}\right)-I_{-}^{\mathrm{S}_{3}}\left(V, V_{\mathrm{bias}}, h^{*}\right)}{I_{+}^{\mathrm{S}_{3}}\left(V, V_{\mathrm{bias}}, h^{*}\right)+I_{-}^{\mathrm{S}_{3}}\left(V, V_{\mathrm{bias}}, h^{*}\right)},
$$

where

$$
\begin{aligned}
I_{\sigma}^{\mathrm{S}_{3}}\left(V, V_{\text {bias }}, h^{*}\right)= & \frac{1}{e R_{\mathrm{p}}} \int d \varepsilon \mathcal{N}^{\mathrm{S}_{3}}\left(\varepsilon+e V_{\text {bias }}\right)\left[f_{\sigma}\left(\varepsilon, V, h^{*}\right)\right. \\
& \left.-f_{0}\left(\varepsilon+e V_{\text {bias }}\right)\right],
\end{aligned}
$$

and $\mathcal{N}_{3}(\varepsilon)$ is the normalized DOS of $\mathrm{S}_{3}$. In the following, we assume for simplicity that $\mathcal{N}^{S_{3}}(\varepsilon)$ is identical to the den- 

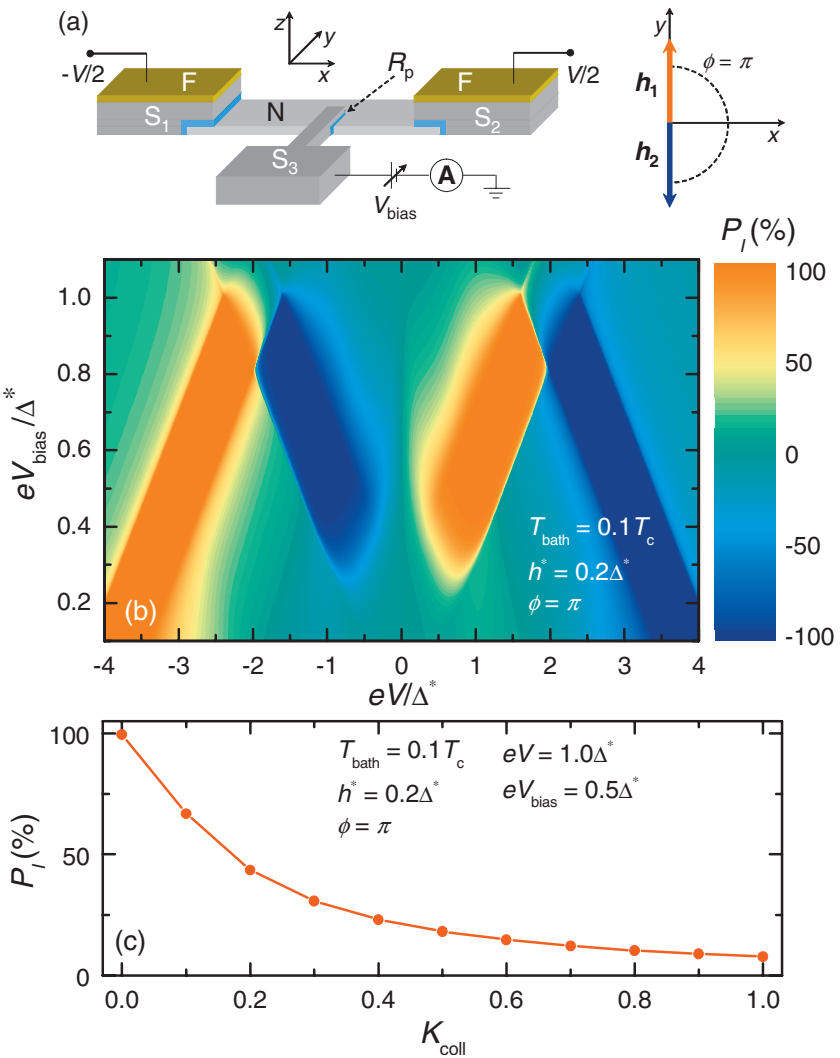

FIG. 14. (Color) (a) Sketch of a possible setup for the implementation of a spin-polarized current source. An additional superconducting electrode $\left(\mathrm{S}_{3}\right)$ is coupled to the $\mathrm{N}$ region through a tunnel junction of resistance $R_{\mathrm{p}}$. The exchange fields in $\mathrm{S}_{1,2}$ are arranged in the antiparallel configuration. Spin-polarized current can be extracted by biasing the $S_{3}$ terminal with $V_{\text {bias. }}$ (b) Contour plot of the nonequilibrium current polarization $P_{I}$ vs $V$ and $V_{\text {bias }}$ at $T_{\text {bath }}=0.1 T_{c}$ and $h^{*}=0.2 \Delta^{*}$. (c) $P_{I}$ vs $\mathcal{K}_{\text {coll }}$ calculated $e V=1.0 \Delta^{*}$ and $e V_{\text {bias }}=0.5 \Delta^{*}$ for $T_{\text {bath }}=0.1 T_{c}, h^{*}=0.2 \Delta^{*}$, and $\phi=\pi$.

sity of states of $S_{1,2}$ in the absence of an exchange field (i.e., $\left.h^{*}=0\right)$.

Figure 14(b) shows the calculated nonequilibrium $P_{J}$ as a function of $V$ and $V_{\text {bias }}$, for $T_{\text {bath }}=0.1 T_{c}$ and $h^{*}=0.2 \Delta^{*}$. For easily attainable values of $V$ and $V_{\text {bias }}$, pure (i.e., $100 \%$ ) spinpolarized current of both the parallel and antiparallel spin species can be achieved. Furthermore, we note that $\left|P_{I}\right|$ largely exceeds $50 \%$ over a wide region in the $\left(V, V_{\text {bias }}\right)$ plane. It is worthwhile to note that replacing the $S_{3}$ terminal with a $\mathrm{N}$ metal reservoir would completely hinder the extraction of a finite spin-polarized current for $\phi=\pi$. This stems from the insensitivity of the tunneling current in a NIN junction to the $\mathrm{N}$-region temperature. The $\phi=0$ case for a $\mathrm{N}$ lead connected to a similar setup was analyzed in Ref. 30.

The role of electron-electron inelastic relaxation is displayed in Fig. 14(c), which shows $P_{I}$ versus $\mathcal{K}_{\text {coll }}$ at $\mathrm{eV}$ $=\Delta^{*}$ and $e V_{\text {bias }}=0.5 \Delta^{*}$, for $T_{\text {bath }}=0.1 T_{c}$ and $h^{*}=0.2 \Delta^{*}$. We find that the suppression of the current polarization occurs on a larger range of values of $\mathcal{K}_{\text {coll }}$ with respect to the electron effective temperature difference [note the different scale for the horizontal axis of Figs. 13(b) and 14(c)]. At $\mathcal{K}_{\text {coll }}=0.1$, for example, $P_{I}$ is reduced only by about $35 \%$. This behavior is not surprising: it originates, as mentioned above, from the fact that the current extracted from $\mathrm{S}_{3}$ is exponentially sensitive to $T_{\sigma}^{\text {eff }}$. As a consequence, even a small temperature difference between spin species yields a large current difference and gives rise to a sizable $P_{I}$ vs $\mathcal{K}_{\text {coll }}$ characteristic.

We shall further comment the impact of relaxation in this setup as compared to that of Fig. 1 by inspecting Figs. 14(c) and 11(b)-11(d). In particular, they show that while electronelectron interaction is crucial in suppressing $P_{I}$ in the former case, it is much less important in the setup of Fig. 1. The reason stems from the fact that while in the present case the electric current is spin dependent only, thanks to the presence of spin-dependent distribution functions in the $\mathrm{N}$ region [as expressed by Eq. (24)], in the setup of Fig. 1, the spin selectivity originates from both the distribution functions $f_{\sigma}$ and the spin-dependent superconducting DOS [see Eqs. (10) and (11)]. From this it follows that, in the first setup, current polarization will completely vanish at quasiequilibrium (where the quasiparticle distribution functions result to be identical and spin independent), while in the second system, spin polarization will persist also for identical thermal distribution functions owing to the additional spin selectivity provided by spin-split DOS in the superconductors.

\section{CONCLUSIONS}

In this paper, we have analyzed the nonequilibrium spindependent transport properties in superconductor-normal metal tunnel nanostructures, where the superconductors present a proximity-induced effective exchange field (equivalent to that of a superconductor in a magnetic field). The latter is due to the proximity of a ferromagnetic thin layer which exerts, under appropriate conditions, a nonlocal influence. We have computed the quasiparticle distributions of a normal metal layer tunnel coupled to two superconductors with noncollinear exchange fields. In the full nonequilibrium limit, i.e., in the absence of any inelastic relaxation mechanisms, the distribution functions depend on the spin and display unusual features, such as population inversion and double-step shape, depending on the bias voltage applied to the superconductors. Spin dependence persists also in the presence of inelastic Coulomb interaction, which produces a smoothing of the sharp features and tends to drive electrons into the quasiequilibrium regime, where the energy relaxation is strong enough to allow electrons to thermalize. This interesting behavior is reflected in the current-voltage characteristic, which shows a strong dependence on the relative orientation of the exchange fields existing in the superconductors. Notably, even in the quasiequilibrium regime, a huge tunnel magnetoresistance ratio and a complete current spin polarization were found over a wide range of bias voltages and for realistic parameters. The impact of the exchange field as well as of the presence of subgap states in the DOS of the superconductors have been addressed.

We have characterized the out-of-equilibrium distribution functions through an "effective temperature" and an "effective chemical potential," defined in an operative sense. We have found that such effective temperatures are strongly spin dependent for antiparallel exchange fields, the relative temperature difference being as high as $500 \%$. Furthermore, we 
have discussed the possibility of producing spin-polarized currents by coupling the $\mathrm{N}$ region to an additional superconducting lead, finding that $100 \%$ spin polarization is realistically achievable. This effect is fairly robust against the occurrence of inelastic electron-electron collisions.

We shall finally comment on some possible applications of the structures presented here. An immediate first application of this system is the implementation of storage cell elements, thanks to the very large TMR values [see Fig. 8(a)]. Magnetic-field-controlled current switches can be envisioned as well [see Fig. 6(a)]. Importantly, power dissipation is intrinsically limited owing to the small currents driven through NIS junctions. For example, assuming $R_{\mathrm{t}}=10^{3} \Omega$ and aluminum (Al) electrodes at $T=0.1 T_{c} \approx 0.12 \mathrm{~K}$, a dissipated power in the range of $10^{-15}-10^{-12} \mathrm{~W}$ can be achieved for $2\left(\Delta^{*}\right.$ $\left.-h^{*}\right) / e<|V|<2 \Delta^{*} / e$. This makes this setup attractive for low-dissipation cryogenic applications. In light of a realistic implementation, ferromagnetic alloys such as $\mathrm{Cu}_{1-x} \mathrm{Ni}_{x}$ (Ref. 53) or $\mathrm{Pd}_{1-x} \mathrm{Ni}_{x}$ (Ref. 54) (which allow fine tuning of $h$ through a proper choice of $x$ ) are promising candidates. For example, in $\mathrm{Pd}_{1-x} \mathrm{Ni}_{x}$ alloy with $x=0.1, h \simeq 10 \mathrm{meV}$ resulting in $\xi_{\mathrm{F}} \approx 5 \mathrm{~nm} .{ }^{54}$ By choosing $\mathrm{Al}$ as $\mathrm{S}$ electrodes [with $\Delta$ $\simeq 200 \mu \mathrm{eV}$ and $\xi_{\mathrm{S}} \approx 300 \mathrm{~nm}$ (Ref. 55)], it turns out that $h^{*}$ in the range $\sim 0.2 \Delta^{*}-0.5 \Delta^{*}$ can be achieved. In such nanostructures, the bias voltage can be fed through outer normal metal electrodes, tunnel coupled to the ferromagnetic layers in order to prevent depolarization of the $\mathrm{F}$ electrodes. This would result in adding in series extra resistances which could be easily engineered in order to minimize the induced correction to both tunnel magnetoresistance and operating voltage.

\section{ACKNOWLEDGMENTS}

Partial financial support from the EU funded HYSWITCH, NanoSciERA "NanoFridge," and RTNNANO projects is acknowledged. *giazotto@sns.it

${ }^{1}$ Theory of Nonequilibrium Superconductivity, edited by N. B. Kopnin (Clarendon, Oxford, 2001).

${ }^{2}$ P. Samuelsson, J. Lantz, V. S. Shumeiko, and G. Wendin, Phys. Rev. B 62, 1319 (2000).

${ }^{3}$ H. Pothier, S. Guéron, N. O. Birge, D. Esteve, and M. H. Devoret, Phys. Rev. Lett. 79, 3490 (1997).

${ }^{4}$ B. J. van Wees, K.-M. H. Lenssen, and C. J. P. M. Harmans, Phys. Rev. B 44, 470 (1991).

${ }^{5}$ P. Samuelsson, V. S. Shumeiko, and G. Wendin, Phys. Rev. B 56, R5763 (1997).

${ }^{6}$ V. S. Shumeiko, G. Wendin, and E. N. Bratus, Phys. Rev. B 48, 13129 (1993).

${ }^{7}$ L. Y. Gorelik, V. S. Shumeiko, R. I. Shekhter, G. Wendin, and M. Jonson, Phys. Rev. Lett. 75, 1162 (1995).

${ }^{8}$ A. F. Volkov, Phys. Rev. Lett. 74, 4730 (1995).

${ }^{9}$ A. F. Volkov and H. Takayanagi, Phys. Rev. B 56, 11184 (1997).

${ }^{10}$ F. K. Wilhelm, G. Schön, and A. D. Zaikin, Phys. Rev. Lett. 81, 1682 (1998).

${ }^{11}$ S. K. Yip, Phys. Rev. B 58, 5803 (1998).

${ }^{12}$ A. F. Morpurgo, T. M. Klapwijk, and B. J. van Wees, Appl. Phys. Lett. 72, 966 (1998).

${ }^{13}$ J. J. A. Baselmans, A. F. Morpurgo, B. J. van Wees, and T. M. Klapwijk, Nature (London) 397, 43 (1999).

${ }^{14}$ F. Giazotto, F. Taddei, T. T. Heikkilä, R. Fazio, and F. Beltram, Appl. Phys. Lett. 83, 2877 (2003).

${ }^{15}$ F. Giazotto, T. T. Heikkilä, F. Taddei, R. Fazio, J. P. Pekola, and F. Beltram, Phys. Rev. Lett. 92, 137001 (2004).

${ }^{16}$ F. Giazotto and J. P. Pekola, J. Appl. Phys. 97, 023908 (2005).

${ }^{17}$ M. A. Laakso, P. Virtanen, F. Giazotto, and T. T. Heikkilä, Phys. Rev. B 75, 094507 (2007).

${ }^{18}$ A. M. Savin, J. P. Pekola, J. T. Flyktman, A. Anthore, and F. Giazotto, Appl. Phys. Lett. 84, 4179 (2004).

${ }^{19}$ J. P. Pekola, F. Giazotto, and O.-P. Saira, Phys. Rev. Lett. 98, 037201 (2007).

${ }^{20}$ O.-P. Saira, M. Meschke, F. Giazotto, A. M. Savin, M. Möttönen, and J. P. Pekola, Phys. Rev. Lett. 99, 027203 (2007).

${ }^{21}$ J. P. Pekola, R. Schoelkopf, and J. Ullom, Phys. Today 57(5), 41 (2004).

${ }^{22}$ F. Giazotto, T. T. Heikkilä, A. Luukanen, A. M. Savin, and J. P. Pekola, Rev. Mod. Phys. 78, 217 (2006).

${ }^{23}$ S. Takahashi, H. Imamura, and S. Maekawa, Phys. Rev. Lett. 82, 3911 (1999).

${ }^{24}$ S. Maekawa, S. Takahashi, and H. Imamura, J. Phys. D 35, 2452 (2002).

${ }^{25}$ Y. Tserkovnyak and A. Brataas, Phys. Rev. B 65, 094517 (2002).

${ }^{26}$ J. Johansson, V. Korenivski, D. B. Haviland, and A. Brataas, Phys. Rev. Lett. 93, 216805 (2004).

${ }^{27}$ S. Takahashi, T. Yamashita, T. Koyama, and S. Maekawa, J. Appl. Phys. 89, 7505 (2001).

${ }^{28}$ I. V. Bobkova and A. M. Bobkov, Phys. Rev. B 74, 220504(R) (2006).

${ }^{29}$ W. Belzig, A. Brataas, Yu. V. Nazarov, and G. E. W. Bauer, Phys. Rev. B 62, 9726 (2000).

${ }^{30}$ F. Giazotto, F. Taddei, R. Fazio, and F. Beltram, Phys. Rev. Lett. 95, 066804 (2005).

${ }^{31}$ F. Giazotto, F. Taddei, R. Fazio, and F. Beltram, Appl. Phys. Lett. 89, 022505 (2006).

${ }^{32}$ See A. I. Buzdin, Rev. Mod. Phys. 77, 935 (2005); F. S. Bergeret, K. B. Efetov, and A. Volkov, ibid. 77, 1321 (2005), and references therein.

${ }^{33}$ F. S. Bergeret, A. F. Volkov, and K. B. Efetov, Phys. Rev. Lett. 86, 3140 (2001).

${ }^{34}$ P. M. Tedrow and R. Meservey, Phys. Rev. Lett. 27, 919 (1971).

${ }^{35}$ R. Meservey and P. M. Tedrow, Phys. Rep. 238, 173 (1994).

${ }^{36}$ J. P. Pekola, T. T. Heikkilä, A. M. Savin, J. T. Flyktman, F. Giazotto, and F. W. J. Hekking, Phys. Rev. Lett. 92, 056804 (2004).

${ }^{37}$ R. C. Dynes, J. P. Garno, G. B. Hertel, and T. P. Orlando, Phys. Rev. Lett. 53, 2437 (1984).

${ }^{38}$ D. R. Heslinga and T. M. Klapwijk, Phys. Rev. B 47, 5157 (1993).

${ }^{39}$ B. L. Altshuler and A. G. Aronov, in Electron-Electron Interac- 
tions in Disordered Systems, edited by A. L. Efros and M. Pollak (Elsevier, Amsterdam, 1985).

${ }^{40}$ A. Kaminski and L. I. Glazman, Phys. Rev. Lett. 86, 2400 (2001)

${ }^{41}$ A. Anthore, F. Pierre, H. Pothier, and D. Esteve, Phys. Rev. Lett. 90, 076806 (2003).

${ }^{42}$ K. E. Nagaev, Phys. Rev. B 52, 4740 (1995).

${ }^{43}$ M. Yu. Kuprianov and V. F. Lukichev, Zh. Eksp. Teor. Fiz. 94, 139 (1988) [Sov. Phys. JETP 67, 1163 (1988)].

${ }^{44}$ B. L. Altshuler and A. G. Aronov, Zh. Eksp. Teor. Fiz. 75, 1610 (1978) [Sov. Phys. JETP 48, 812 (1978)].

${ }^{45}$ A. Kamenev and A. Andreev, Phys. Rev. B 60, 2218 (1999).

${ }^{46}$ B. Huard, A. Anthore, F. Pierre, H. Pothier, N. O. Birge, and D. Esteve, Solid State Commun. 131, 599 (2004).

${ }^{47}$ M. Yu. Kupriyanov, A. Brinkman, A. A. Golubov, M. Siegel, and H. Rogalla, Physica C 326-327, 16 (1999).
${ }^{48}$ M. Tinkham, Introduction to Superconductivity (Dover, New York, 1996).

${ }^{49}$ F. J. Jedema, A. T. Filip, and B. J. van Wees, Nature (London) 410, 345 (2001).

${ }^{50}$ M. Johnson, Phys. Rev. Lett. 70, 2142 (1993).

${ }^{51}$ T. T. Heikkilä, Ph.D. thesis, HUT Helsinki, 2002.

${ }^{52}$ The constraint $R_{p} \gg R_{t}$ ensures the nonequilibrium condition in the $\mathrm{N}$ region not to be appreciably perturbed by the current extraction from the superconducting terminal $\mathrm{S}_{3}$.

${ }^{53}$ V. V. Ryazanov, V. A. Oboznov, A. Yu. Rusanov, A. V. Veretennikov, A. A. Golubov, and J. Aarts, Phys. Rev. Lett. 86, 2427 (2001).

${ }^{54}$ T. Kontos, M. Aprili, J. Lesueur, and X. Grison, Phys. Rev. Lett. 86, 304 (2001).

${ }^{55}$ J. Romijn, T. M. Klapwijk, M. J. Renne, and J. E. Mooij, Phys. Rev. B 26, 3648 (1982). 